UC-25: Metallurgy and Ceramics TID-4500 (15th Ed.)

\title{
THE DIFFUSION OF KRYPTON-85 FROM URANIUM DIOXIDE POWDER
}

A. B. Auskern

February 1960

Contract AT-11-1-GEN-14

Price $\$ .50$

Available from the Office of Technical Services,

Department of Commerce,

Washington 25, D. C.

NOTE

This document is an interim memorandum prepared primarily for internal reference and does not represent a final expression of the opinion of Westinghouse. When this memorandum is distributed externally, it is with the express understanding that Westinghouse makes no representation as to completeness, accuracy, or usability of information contained therein.

\author{
BETTIS ATOMIC POWER LABORATORY \\ PITTSBURGH, PENNSYLVANIA \\ OPERATED FOR THE U. S. ATOMIC ENERGY COMMISSION BY \\ WESTINGHOUSE ELECTRIC CORPORATION
}


SPECIAL EXTERNAL DISTRIBUTION

Manager, P1tsburgh Naval Reactors Operatıons Office, AEC

This report was prepared as an account of Government sponsored work. Heither the United States, nor the Commission, nor any person acting on behalf of the Commission:

A. Makes any warranty or representation, expressed or implied, with respect to the accuracy, completeness, or usefulness of the information contained in this report, or that the use of any information, apparatus, method, or process disclosed in this report may not infringe privately owned rights; or

B. Assumes any liabilities with respect to the use of, or for damages resulting from the use of any information, apparatus, method, or process disciosed in this report.

As used in the above, "person acting on behalf of the commission" includes any employe or contractor of the Commission, or employe of such contractor, to the extent that such employe or contractor of the commission, or employe of such contractor prepares, disseminates, or provides access to, any information pursuant to his employment or contract with the Commission, or his employment with such contractor. 
Page No.

I. INTRODUCTION

II. EXPERIMENTAL

A. Powders Investigated

3

B. Irradiation of Samples

C. Diffusion Anneals

D. Analytical Procedure

5

III. RESULTS

5

IV. DISCUSSION

8

REFERENCES 
-

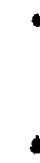




\begin{abstract}
The diffusion of $\mathrm{Kr}^{85}$ in two $\mathrm{UO}_{2}$ powders from different sources has been studied by performing a series of post-irradiation anneals on the powders. The emanation data were analyzed in a manner that considered the effect of sintering as well as the effect of a distribution of farticle sizes within the sample Measurements were made in the temperature range of 900 to $1500^{\circ} \mathrm{C}$. The time at a temperature was between 8 and 24 hours The diffusion coefficlents for $K r^{85}$ in the two powders can be represented by the equations. $D=265 \times 10^{-4}$ exp $-65,500 / R T$ for $\mathrm{UO}_{2}$ prepared from crushed $\mathrm{UO}_{2}$ pellets and, for a chemically prepared $\mathrm{UO}_{2}$ powder, $D=49 \times 10^{-4}$ exp $-73,800 / R T$.
\end{abstract}

\title{
THE DIFFUSION OF KRYPTON-85 FROM URANIUM DIOXIDE POWDER
}

\author{
A. B. Auskern
}

\section{INTRODUCTION}

The present investigation forms part of a larger program concerned with the release of fission gases from uranium dioxide. In this study the release of krypton-85 from powdered $\mathrm{UO}_{2}$ was considered. Experiments were performed to determine the kinetics and temperature dependence of the release process.

Extensive discussions of the work done in this area are presented in reviews by Lustman (Ref 1 ) and by Eichenberg, et al. (Ref 2). Evidence indicates that the release of fission gases $\mathrm{from}^{\mathrm{U}} \mathrm{UO}_{2}$ is one of diffusion through the lattice. The measurements of Booth and Rymer (Ref 3) on powders prepared from fused $\mathrm{UO}_{2}$ give some support for this mechanism. They showed that the release of xenon-133, in agreement with an appropriate solution to the diffusion equation and within the limitations of the experiment, varied directly as the reciprocal of the particle radius, and directly as the square root of the annealing time. For this powder, the diffusion coefficient variation for xenon-133 from 1000 to $1500^{\circ} \mathrm{C}$ is represented by the equation $\mathrm{D}=1.5 \times 10^{-8} \exp -46,000 / \mathrm{RT}$. Booth and Rymer also reported that there was no significant difference between xenon and krypton diffusion rates.

Post-irradiation diffusion anneals on dense $\mathrm{UO}_{2}$ sintered pellets have been performed (Ref 4 ) in the temperature range of 1000 to $1575^{\circ} \mathrm{C}$. Diffusion coefficients varied from about $3.5 \times 10^{-20}$ at $1000^{\circ} \mathrm{C}$ to $1 \times 10^{-16} \mathrm{~cm}^{2} / \mathrm{sec}$ at $1575^{\circ} \mathrm{C}$. The activation energy calculated from the results is $58.5 \mathrm{kcal} / \mathrm{mole}$. For this work the absolute value for the diffusion coefficient is in some doubt because of the difficulty in assigning an average spherical "radius" to a sintered pellet.

A series of experiments has been performed to measure the solubility and diffusion of helium in $\mathrm{UO}_{2}$ powder (Refs 2 and 4). The helium diffusion data fit the spherical diffusion model very well. Diffusion coefficients ranging from $1 \times 10^{-17} \mathrm{~cm}^{2} / \mathrm{sec}$ at $700^{\circ} \mathrm{C}$ to $2 \times 10^{-15} \mathrm{~cm}^{2} / \mathrm{sec}$ at $1000^{\circ} \mathrm{C} \mathrm{were}$ calculated. The activation energy is $46 \mathrm{kcal} / \mathrm{mole}$, the same as that found by Booth and Rymer (Ref 3 ) for xenon. Diffusion of the helium is, however, at least an order of magnitude faster than the xenon diffusion. 
Recent work by Lindner and Matzke (Refs 5 and 6) on xenon diffusion from uranium oxide powder has been published. They report in their initial study, with $\mathrm{UO}_{2.0}$ and $\mathrm{UO}_{2.67}$ powders in the temperature range of 500 to $1100^{\circ} \mathrm{C}$ for relatively short periods of time, an activation energy of about $34 \mathrm{kcal}$ for $\mathrm{UO}_{2}$ and somewhat less for $\mathrm{UO}_{2.67^{\circ}}$ Diffusion in $\mathrm{UO}_{2.67^{\prime}}$, however, is about two orders of magnitude faster than in $\mathrm{UO}_{2}$. In a second study which concerned the effect of $\mathrm{O} / \mathrm{U}$ ratio on the diffusion of xenon, the activation energy and diffusion coefficients found with $\mathrm{UO}_{2} .00$ were very similar to those of Booth and Rymer (Ref 3). About an order of magnitude increase in $D$ occurred when the oxygen content was raised from $\mathrm{UO}_{2.00}$ to $\mathrm{UO}_{2.02}$, and from $\mathrm{UO}_{2.02}$ to $\mathrm{UO}_{2.12^{\circ}}$

The Booth and Rymer work is most similar to the present investigation. Some data from Booth and Rymer will be discussed in detail for their general applicability. For the calculation of diffusion coefficients in the fused $\mathrm{UO}_{2}$ powder, a particle radius of 28 microns was used. This is the average radius given by the screen fraction used, which was -200 to +325 mesh. However, the assumption that an average screen radius represents the true particle radius can be misleading. It has been shown that the spherical radius when calculated from surface area and density measurements is smaller than that given by sieve sizes. * For example, for a powder prepared from fused $\mathrm{UO}_{2}$ (liquid density, $10.95 \mathrm{~g} / \mathrm{cc}$ ) and screened between 80 and 120 mesh screens, the surface area and density measurements resulted in an average spherical radius of 15 microns. However, the average radius for the screen fraction is 75 microns. Since such a difference may be present in the powders studied by Booth and Rymer, then their diffusion coefficients can be too large.

The Booth and Rymer report contains much information on the amount of gas released from sintered compacts. For a given compact type, the fraction of xenon released varies inversely as the compact density. However, for compacts of the same density, but from different sources, there are large differences in the amount of gas released. What is surprising about these data is the number of samples which gave release rates much greater than that for the fused powder. For the fused powder, for example, $2.1 \%$ xenon diffused out at $1400^{\circ} \mathrm{C}$ in three hours. For some low density pellets ( $85 \%$ theoretical), the fraction that diffused out under identical experimental conditions was much higher-ranging from 15 to $35 \%$. If, as it is assumed, the rate controlling step is diffusion through the lattice and the diffusion coefficient of xenon through the $\mathrm{UO}_{2}$ lattice is independent of the powder source or treatment, then, in order for the gas release to be greater in the compact, the diffusion path length in the compact must be smaller than that in the fused powder. This further implies that the surface areas of these compacts are greater than that of the fused -200 to +325 mesh $\mathrm{UO}_{2}$ powder. Of course, the rate controlling step for diffusion from sintered compacts containing some open porosity may not be pure lattice diffusion. If this is the case, then the greater release rate for some compacts may be reasonable if the data are analyzed with some other appropriate model. Unfortunately, Booth and Rymer report no surface area measurements which might help clear up some of the apparent discrepancies between the powder and compact data.

The absolute magnitude of diffusion coefficients is very sensitive to the particle radius used. For powders like $\mathrm{UO}_{2}$ which can be porous, highly irregular in shape, and which usually consist of groups of fine crystallites, the use of microscopic measurements or sieve screen estimates for particle radii is unrealistic because much of the available surface may not be directly visible. Flugge and Zimens (Ref 7), in their paper on the Hahn emanation method, consider the surface as measured by gas adsorption to be the best estimate of an emanating surface.

With fine powders undergoing anneals at high temperatures, the possibility that sintering can occur and reduce the powder surface area must be considered. The occurrence of sintering during

*A. B. Auskern, unpublished data, Bettis Laboratory. 
an anneal makes the effective spherical radius of the powder larger and results in the emanation rate being reduced. Either of two things is essential then to make emanation data meaningful: the powder must be prepared in such a way that it will undergo no surface area change during an anneal or, if this is difficult, account must be taken of the surface area change in order to correctly evaluate the diffusion coefficients.

Neglecting sintering results in calculated diffusion coefficients that are too small. Also, if sintering occurs over a range of temperatures, the constantly changing particle radius indicates that the activation energy for the process would be higher than that found if the effect of sintering were neglected.

A further consideration is that the sample does not consist of a single size particle, but varies over a range of sizes. During an anneal the fine particles become depleted more rapidly than the coarse, and the emanation kinetics deviate from the ideal case where a single uniform particle size is assumed.

In this study two powders from sources other than fused $\mathrm{UO}_{2}$ were investigated. The analysis of the emanation rate data included the effect of sintering and depletion of fine particles.

\section{EXPERIMENTAL}

A. Powders Investigated

Two uranium dioxide powders were used in this study. The powder designated NUR was prepared by the conversion of uranyl nitrate hexahydrate to $\mathrm{UO}_{3}$, and then the $\mathrm{UO}_{3}$ to the dioxide by heating in hydrogen at $800^{\circ} \mathrm{C}$. The $\mathrm{UO}_{2}$ formed was annealed in hydrogen at $1650^{\circ} \mathrm{C}$ for 21 hours, and then ball milled for 16 hours in a rubber lined mill using uranium balls. The surface area of this powder, as determined by gas adsorption, is $1.3 \mathrm{~m}^{2} / \mathrm{g}$ and the liquid density, $10.71 \mathrm{~g} / \mathrm{cc}$. Chemical analysis showed the material to be $\mathrm{UO}_{2.002}$ and the impurity level, estimated by spectrographic analysis, to be $0.02 \%$.

The second powder studied was prepared by crushing a number of sintered $\mathrm{UO}_{2}$ pellets $192 \%$ theoretical density) and collecting the -200 to +325 mesh screen fraction. The surface area of this powder is $0.1 \mathrm{~m}^{2} / \mathrm{g}$ and the density is $10.67 \mathrm{~g} / \mathrm{cc}$. The material was analyzed as $\mathrm{UO}_{2.004}$ Spec- $^{\circ}$ trographic analysis gave an impurity content of about $0.05 \%$, with the principal impurity being iron.

The average spherical radii of the powders were calculated from surface area and density measurements. The calculation for the spherical radius involves the assumptions that the particles are perfect spheres and are of uniform size. The relationship

$$
a=\frac{3}{(s)(d)}
$$

is used, where "a" is the average particle radius in $\mathrm{cm}, \mathrm{s}$ is the surface area $\left(\mathrm{cm}^{2} / \mathrm{g}\right)$, and $\mathrm{d}$ is the density $(\mathrm{g} / \mathrm{cc})$. For the NUR powder, $\mathrm{a}=0.22$ microns; for the crushed $\mathrm{UO}_{2}, \mathrm{a}=2.8 \mathrm{microns}$.

B. Irradiation of Samples

Irradiations of the samples were carried out at the Brookhaven National Laboratory reactor. Reactor hole N-5 was used for all irradiations. This is an air cooled facility (ambient temperature about $50^{\circ} \mathrm{C}$ ) designed to permit sample insertion and withdrawal while the reactor is in full operation.

The uranium dioxide samples, about three grams in weight, were contained in quartz vials under a helium atmosphere. The samples were irradiated to an nvt of about $4 \times 10^{16}$ thermal neutrons $/ \mathrm{cm}^{2}$. This resulted in an approximate burnup of $0.004 \%$ of $\mathrm{U}^{235}$ atoms present, and produced about $3 \times 10^{12} \mathrm{Kr}^{85}$ atoms in the three gram sample.

The amount of $\mathrm{Kr}^{85}$ actually produced in the uranium dioxide during irradiation was determined by total dissolution of a sample in concentrated nitric acid and collection of the released fission gases. 
In all cases the quantity of $\mathrm{Kr}^{85}$ measured was less than the amount calculated from the conditions of the irradiation. For the NUR powder the amount of $\mathrm{Kr}^{85}$ determined was about $65 \%$ of that calculated; for the crushed $\mathrm{UO}_{2}, 52 \%$ of the amount calculated was found. These differences may be the result of errors in neutron flux estimates and the recoil of fission fragments from the $\mathrm{UO}_{2}$ powder during irradiation.

A diagram of the dissolving and gas collection system is shown in Fig. 1. In operation, a flow of helium gas is established through the complete system, including the activated charcoal traps. The crucible containing the $\mathrm{UO}_{2}$ sample is upset with a magnetic stirring rod and the rapid $\mathrm{UO}_{2}-\mathrm{HNO}_{3}$ reaction begins. The acid is frequently agitated and then boiled gently for about 15 to 20 minutes to facilitate complete removal of the gases. The ferrous sulfate solution in the train is used to remove nitric oxide and nitrogen dioxide from the gas stream. Finally, the charcoal traps are isolated, evacuated, and sealed off directly above the charcoal. In no case was any $\mathrm{Kr}^{85}$ detected in the second charcoal trap.

C. Diffusion Anneals

A drawing of the annealing system is shown in Fig. 2. Emanation data over the complete temperature range are obtained without changing the sample. With the system evacuated, the sample is suspended above the hot zone and the furnace brought to the lowest annealing temperature. The system is then isolated from the vacuum station and the sample is lowered into the hot zone. After heating for the desired length of time, the sample is raised from the hot zone and the gases evolved during the anneal are collected on activated charcoal cooled with liquid nitrogen. Usually, for anneals up to $1100^{\circ} \mathrm{C}$, there is enough degassing of the furnace and $\mathrm{UO}_{2}$ powder to raise the system pressure to around 500 microns. By following the decrease in the pressure during gas collection, the point at which essentially all the gas is collected is easily determined. At this point the sampling bulb is sealed off. The sample is then lowered for the next anneal at the same temperature.

With the sample raised out of the hot zone, the furnace temperature is raised to that required for the next anneal. Anneals at $100^{\circ} \mathrm{C}$ increments were made up to $1400^{\circ} \mathrm{C}$ for the NUR powder and up to $1500^{\circ} \mathrm{C}$ for the crushed $\mathrm{UO}_{2}$ powder. The total time at a temperature was between 8 and 24 hours; three or four gas samples were collected during this time.

The furnace was controlled with a Bristol-Pyrovane temperature controller to within $\pm 5^{\circ} \mathrm{C}$, and temperatures were read with a $\mathrm{Pt}-\mathrm{Pt}, 10 \mathrm{Rh}$ thermocouple located outside the combustion tube at the sample level.

Separate anneals were made on unirradiated samples of the two powders to estimate the amount of sintering that could occur during an emanation anneal. The anneals were performed in hydrogen at $1400^{\circ} \mathrm{C}$ for 22 hours. The results of surface area measurements showed a decrease, by a factor

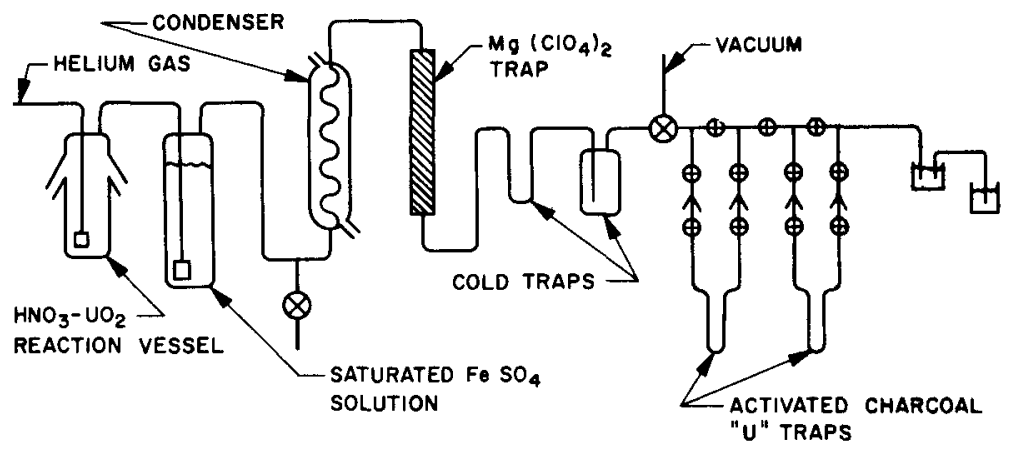

Fig. I Total Dissolution System 


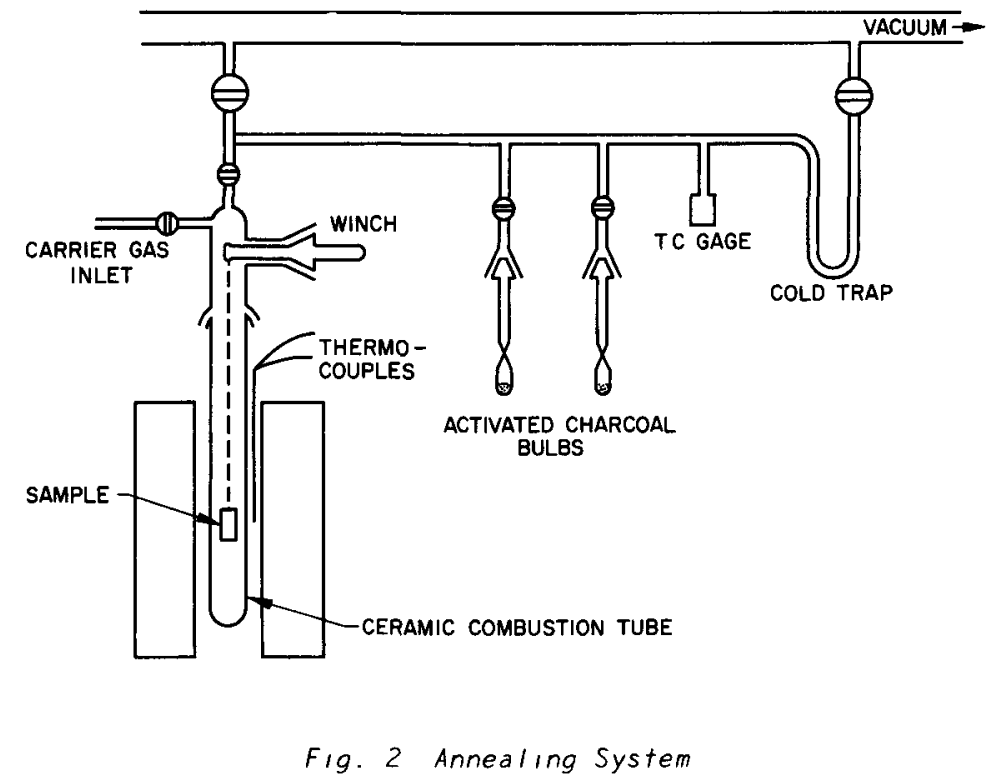

of 5, for both powders. Thus, the spherical radii of the powders can be estimated to have increased by roughly the same factor (density changes have been neglected). The sintered radius of the crushed $\mathrm{UO}_{2}$ powder is then about 14 microns, and that of the NUR powder, about 1 micron.

D. Analytical Procedure

The bulbs containing the collected gases were analyzed for $\mathrm{Kr}^{-85}$ content by gamma ray spectroscopy methods. A spectrum profile in the vicinity of $0.52 \mathrm{Mev}$ was obtained with a 100 -channel analyzer employing a thallium-activated KI well-type crystal. A peak of this energy is characteristic of the decay scheme involving $\mathrm{Kr}^{85}$. The sample activity at this energy may be related, with consideration for the counter efficiency (approximately $9 \%$ ) and the branching ratio $\left(6.5 \times 10^{-3}\right.$ ), to the number of $\mathrm{Kr}^{85}$ atoms present. The experiments were completed within a few months after the sample was irradiated. Since krypton-85 has a 10.3 year half-life, no corrections for decay were made.

The counting for the total dissolution experiments was handled in the same manner, except that the " $U$ " tubes containing the fission gases were counted using a flat 3 -in. diam crystal.

\section{RESULTS}

The data for the complete heat treatment of a powder can be plotted as the cumulative fractional release vs the cumulative time. Discontinuities in the curve result when the temperature is raised. These plots for the two uranium dioxide powders are shown in Fig. 3 .

For the calculation of diffusion coefficients, the model assumed is that of diffusion from a group of uniform spheres at some initial $\mathrm{Kr}^{85}$ concentration, with the surface maintained at zero concentration. A solution to the diffusion equation (Ref 4 ) for this case is

$$
F=1-\frac{6}{\pi^{2}} \sum_{n=1}^{\infty} \frac{1}{n^{2}} e^{\frac{-n^{2} \pi^{2} D t}{a^{2}}} \text {, }
$$




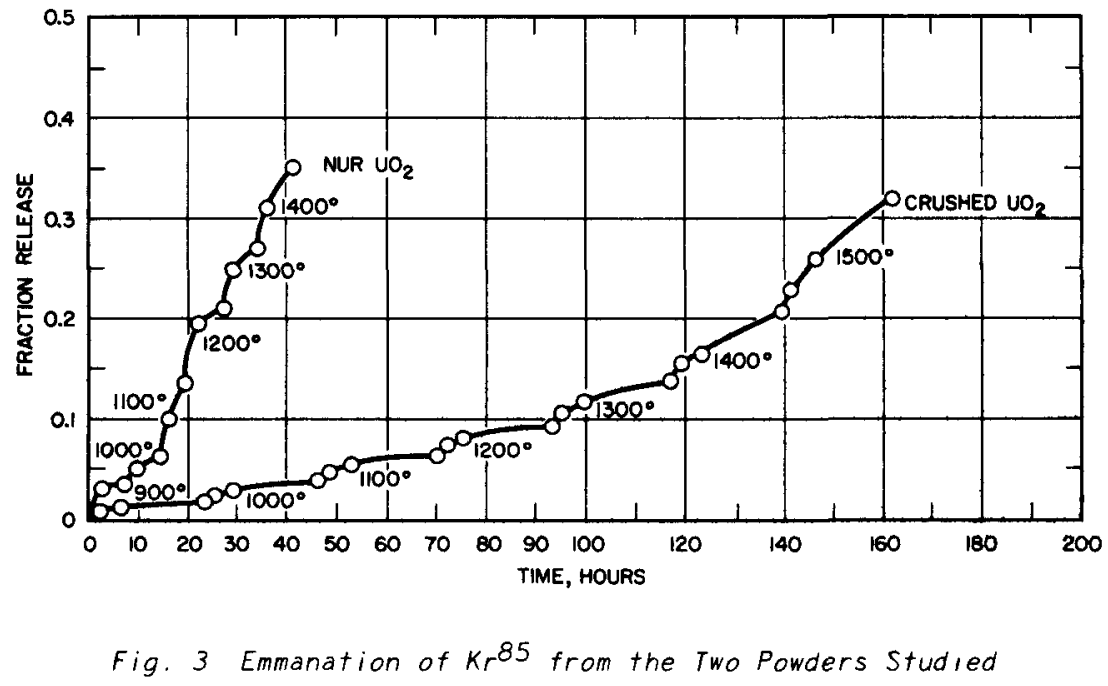

where

$\mathbf{F}=$ fractional release,

$\mathrm{D}=$ diffusion coefficient, $\mathrm{cm}^{2} / \mathrm{sec}$,

$\mathrm{t}$ = time, sec, and

$\mathrm{a}=$ particle radius, $\mathrm{cm}$.

For short times, an approximation*

$$
F=\frac{6}{v \sqrt{\pi}}+\frac{3}{v^{2}},
$$

where $v^{2}=\left(a^{2} / D t\right)$, may be used.

In calculating diffusion coefficients at different temperatures, account must be taken of the experimental condition that the zero time for the anneal is not known for all temperatures after the first. Each isothermal portion of the cumulative fractional release vs cumulative time curve represents only the measured segment of a hypothetical continuous anneal which started at some undefined zero time. Thus, the equations just listed are directly applicable only to the first anneal, since this is the only anneal to which the boundary conditions of the diffusion equation solution directly apply. However, diffusion coefficients at temperatures above the first are readily determined.

From a master plot of $\mathrm{Dt} / \mathrm{a}^{2}$ vs $\mathrm{F}$, values of $\mathrm{Dt} / \mathrm{a}^{2}$ for the fractional release obtained during an anneal are read. These values are plotted against time. Since the zero time is not known, only the time differences between samples at a particular temperature can be plotted, with the initial point (equivalent to the last point at the previous lower temperature) set at an arbitrary position on the time scale. The relationship between $\mathrm{Dt} / \mathrm{a}^{2}$ and $\mathrm{t}$ is linear, and the slope of the line through the experimental points is $\mathrm{D} / \mathrm{a}^{2}$. From a knowledge of "a," $\mathrm{D}$ is calculated directly. Extrapolating the curve to $\mathrm{Dt} / \mathrm{a}^{2}=0$ fixes a theoretical zero time. The significance of the theoretical zero time is that it is the amount of time at temperature the sample would take to reach the concentration distribution defined by the first experimental point, assuming that the concentration in the sample was uniform at $\mathrm{t}=0$.

The effects of a changing particle radius, as well as depletion of $\mathrm{Kr}^{85}$ in the fine particles, must be evaluated. The occurrence of either or both conditions changes the shape of the emanation curve.

*J. M. Markowitz, personal communication, Bettis Laboratory. 
Consider first that sintering is occurring. During the anneal the spherical radius increases from its initial value to some final value at the end of the anneal. Thus, the rate of emanation of the gas does not follow a specific diffusion curve. At the start of the anneal, emanation follows the curve calculated with the initial small radius. However, as sintering progresses and the particles become larger, the emanation rate slows down and leaves the initial rate curve, passing through a family of rate curves (each corresponding to a larger particle size). For long enough times, the emanation rate curve approaches the curve given by the final particle size. Thus, a true diffusion curve does not result. The emanation data lie between the curves defined by the initial and final particle sizes.

When plotting the data as $\mathrm{Dt} / \mathrm{a}^{2}$ vs $\mathrm{t}$ to calculate diffusion coefficients, a similar type of curve is observed. Had no sintering taken place, the $D t / a^{2}$ vs $t$ curve would be a straight line of slope $\mathrm{D} / \mathrm{a}^{2}$. With the occurrence of sintering, the experimental curve moves from the initial slope, crosses a family of lines, each corresponding to a larger "a" value (and smaller slope), and for long enough times, parallels the line, the slope of which is given by the final sintered radius. Thus, the experimental $\mathrm{Dt} / \mathrm{a}^{2}$ vs $\mathrm{t}$ curve is a curved line lying between the lines defined by the initial and final particle radius. The calculation of the diffusion coefficient must then be made from the initial slope of the $\mathrm{Dt} / \mathrm{a}^{2} \mathrm{vs} \mathrm{t}$ curve since, for this region at least, a reasonable value for the particle radius is known.

An analagous set of curves results when emanation from a range of particle sizes is considered. Initially, the emanation rate is rapid because of diffusion from the fine particles. As the amount of gas in these particles becomes depleted, the rate slows down and becomes controlled by emanation from the larger particles. Thus, the resulting $D t / \mathrm{a}^{2}$ vs $t$ plot is also not linear. It is a curved line similar to that resulting from sintering. The initial slope is steep, corresponding to emanation from the finer fractions. As these particles become depleted, the slope becomes smaller and smaller and approaches finally the slope given by the limiting emanating particles.

The situation is complex when sintering and depletion are occurring together. Detailed knowledge of the rate of sintering is required to estimate the combined effects, as well as information on the initial size distribution and the manner in which the distribution changes as a function of time and temperature.

The shape of an experimental emanation curve is usually modified by both sintering and particle depletion. As in the case of sintering, the best estimate for the diffusion coefficient is given by the initial slope of the $\mathrm{Dt} / \mathrm{a}^{2}$ vs $\mathrm{t}$ curve and the initial particle radius. If the particle size distribution of the sample contains many very fine particles and these control the initial rate of emanation, then the diffusion coefficient calculated using the average particle radius will be too large. It would be difficult to correct this without knowing the particle size distribution.

Since the emanation data in the experiments performed here were obtained from one sample for each powder for the whole temperature range, the value of the particle radius must be constantly revised at each temperature to account for particle sintering and depletion.

Both the diffusion coefficient and the new number for the particle radius are determined from the experimental Dt/a ${ }^{2}$ vs $t$ curve. Starting with the lowest annealing temperature, the first diffusion coefficient is calculated from the initial slope and the known initial particle radius. With this diffusion coefficient and the slope observed at the end of the anneal, a value for "a" can be calculated. This "a" value is not necessarily the average particle radius at the end of the anneal; it is a fictitious radius which refers only to the condition of the material that is emanating at the end of the anneal and includes the effects of sintering as well as depletion of the fine particles. This radius is then used for the calculation of the diffusion coefficient for the next highest temperature anneal. The calculation is repeated again and another "a" value is determined from the slope at the end of the higher temperature anneal. The data from the anneals of the two uranium dioxide powders have been treated 
TABLE I

DIFFUSION COEFFICIENTS OF $\mathrm{Kr}^{85}$ IN UO $_{2}$

\begin{tabular}{|c|c|c|}
\hline \multirow[b]{2}{*}{ Temperature $\left({ }^{\circ} \mathrm{C}\right)$} & \multicolumn{2}{|c|}{ Diffusion Coefficient $\left(\mathrm{cm}^{2} / \mathrm{sec}\right)$} \\
\hline & Crushed $\mathrm{UO}_{2}$ & $\mathrm{NUR} \mathrm{UO}_{2}$ \\
\hline 900 & $6.7 \times 10^{-17}$ & $3.9 \times 10^{-18}$ \\
\hline 1000 & $1.3 \times 10^{-15}$ & $5.3 \times 10^{-17}$ \\
\hline 1100 & $8.3 \times 10^{-15}$ & $7.6 \times 10^{-16}$ \\
\hline 1200 & $6.6 \times 10^{-14}$ & $5.3 \times 10^{-15}$ \\
\hline 1300 & $2.2 \times 10^{-13}$ & $1.8 \times 10^{-14}$ \\
\hline 1400 & $5.9 \times 10^{-13}$ & $1.0 \times 10^{-13}$ \\
\hline 1500 & $2.2 \times 10^{-12}$ & - \\
\hline
\end{tabular}

in this manner. Diffusion coefficients have been calculated at the various temperatures and are listed in Table I.

At temperatures above $1200^{\circ} \mathrm{C}$ for the crushed $\mathrm{UO}_{2}$, the $\mathrm{Dt} / \mathrm{a}^{2}$ vs $\mathrm{t}$ curves were linear for the duration of the anneals. For these higher temperatures no further adjustment of "a" was required. For the NUR powder a continuous adjustment of "a" was necessary, although at the higher temperatures the amount of change became less and less.

The variation of $\log \mathrm{D}$ with $1 / \mathrm{T}$ is shown in Fig. 4. The variation is quite linear over the whole temperature range investigated, with no breaks noted in the curve. The diffusion coefficients for the crushed $\mathrm{UO}_{2}$ powder are about an order of magnitude higher than those for the NUR powder. The slopes of the curves correspond to an activation energy of $65.5 \mathrm{kcal} / \mathrm{mole}$ for the crushed powder, and $73.8 \mathrm{kcal} / \mathrm{mole}$ for the NUR powder. The variation in $\mathrm{D}$ with temperature can be represented by the equations $\mathrm{D}=2.65 \times 10^{-4} \exp -65,500 / \mathrm{RT}$ for crushed $\mathrm{UO}_{2}$, and $\mathrm{D}=4.9 \times 10^{-4} \exp -73,800 / \mathrm{RT}$ for the NUR powder.

\section{DISCUSSION}

A comparison should be made between the " $a$ " values of the powders after the sintering anneals and the final " $a$ " values that were calculated from the analysis. These are given in the following table.

\section{Powder \\ NUR}

Crushed

$\frac{\text { Radius }}{0.22 \text { microns }} \quad \frac{\text { After Sintering }}{1 \text { micron }}$

14
Final Emanation Radius

7 microns*
$43 * *$

* This radius is still changing at $1400^{\circ} \mathrm{C}$.

* No further change after $1200^{\circ} \mathrm{C}$.

These numbers should not be the same. The radius calculated from the data is based on an emanation curve which is weighted (because of depletion of the fine particles) toward the coarser particle fraction. The radius after the sintering anneals is determined by a gas adsorption method and includes all the particles, fines as well as coarse. Thus, this radius should be smaller than the emanation radius and this is observed. For the crushed $\mathrm{UO}_{2}$, the sintered radius is 14 microns and 


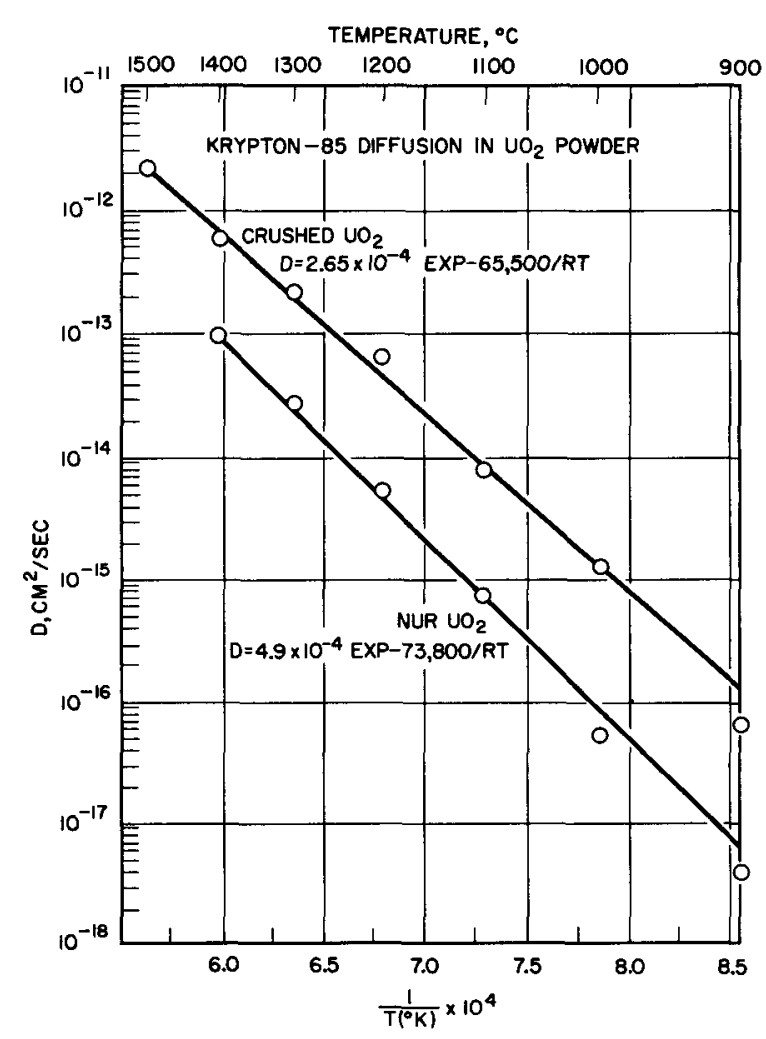

Fig. 4 Temperature Dependence of the Diffusion Coefficient

been about $0.04 \%$. Because of the short neutron exposure the samples received, a quantity of $\mathrm{Kr}{ }^{85}$ corresponding to $0.04 \%$ of the total could not have been readily detected.

The results found here for $\mathrm{Kr}^{85}$ diffusion are among the highest that have been reported for rare gases in $\mathrm{UO}_{2} \cdot$ The diffusion coefficients are significantly higher than those reported for UO $\mathrm{UO}_{2}$ com pacts (Ref 4), and the ones given by Booth and Rymer (Ref 3), and by Lindner and Matzke (Refs 5 and 6) for xenon in $\mathrm{UO}_{2}$. At 900 and $1000^{\circ} \mathrm{C}$, helium diffusion coefficients are greater than those found for $\mathrm{Kr}^{85}$, but the activation energy is lower (Ref 4 ).

The results of thoron diffusion experiments in $\mathrm{UO}_{2}$, using the Hahn emanation technique, give estimated diffusion coefficients and activation energies in the same range as those found here. In the work of Anderson, et al. (Ref 8), an activation energy of $81 \mathrm{kcal} / \mathrm{mole}$ was found above $1150^{\circ} \mathrm{C}$. The process responsible for this activation energy is considered to be bulk diffusion through the lattice. A diffusion coefficient estimated from the emanation data is about $10^{-14} \mathrm{~cm}^{2} / \mathrm{sec} \mathrm{at} 1200^{\circ} \mathrm{C}$. Recent work with the Hahn emanation method on $\mathrm{UO}_{2}$ compacts gives activation energies between 60 and $85 \mathrm{kcal} / \mathrm{mole}$ above $1100^{\circ} \mathrm{C}$. * Estimated diffusion coefficients are around $10^{-14} \mathrm{~cm}^{2} / \mathrm{sec}^{-1}$ $1400^{\circ} \mathrm{C}$.

In the temperature range investigated, diffusion rates for the crushed $\mathrm{UO}_{2}$ are about an order of magnitude greater than those of the NUR powder. Since no provisions were made for the maintenance of a reducing atmosphere during the anneals, the possibility exists that some oxidation can take place and result in enhanced diffusion coefficients for the crushed $\mathrm{UO}_{2}$ powder. Lindner and Matzke (Ref 6) have shown that very slight amounts of excess oxygen significantly increase the xenon diffusion

\footnotetext{
*J. C. Clayton, personal communication, Bettis Laboratory.
} 
rate. A similar effect was also observed for oxygen diffusion in $\mathrm{UO}_{2}$, * especially for nearly stoichiometric compositions.

Further work is needed before the mechanism of fission gas release can be precisely described. For example, the effect of burnup has not been evaluated. In these experiments the powders received a short reactor exposure compared to the exposure for some pellet work. Also, in the work performed here and in the referenced works, the annealing times were relatively short, i.e., less than 24 hours. Bostrom* has demonstrated that, for dense $\mathrm{UO}_{2}$ plates, the emanation rate for xenon for the first 10 to 30 hours usually corresponds to a diffusion coefficient greater than that observed for the final 30 to about 120 hours. This may be associated with a "burst" release that has been observed on initially heating sintered $\mathrm{UO}_{2}$ materials. For the emanation curves in the experiments reported here, a model of pure diffusion was assumed, complicated by particle sintering and depletion. However, the possibility that "bursts" occurred cannot be excluded, especially from the data for the low temperature anneals.

The question of a low-temperature, low activation energy release process for fission gas in $\mathrm{UO}_{2}$ has not been resolved. It was not observed in this study nor in the work of Booth and Rymer (Ref 3) or Lindner and Matzke (Ref 6). However, work with the Hahn emanation method and with gas release from sintered pellets indicates that a different diffusion mechanism may be operative at lower temperatures.

\section{REFERENCES}

1. B. Lustman, "Release of Fission Gases from UO 2 ," WAPD-173 (March 1957).

2. J. D. Eichenberg, P. W. Frank, T. J. Kisiel, B. Lustman, and K. H. Vogel, "Effects of Irradiation on Bulk $\mathrm{UO}_{2}$," in "Fuel Elements Conference, Paris, November 18-23, 1957," TID-7546, Book 2, pp 616-716.

3. A. H. Booth and C. T. Rymer, "Determination of the Diffusion Constant of Fission Zenon in $\mathrm{UO}_{2}$ Crystals and Sintered Compacts," CRDC-720 (1958).

4. J. Belle, "Properties of Uranium Dioxide," Geneva Conference Paper, A/Conf. 15/P/2404 (1958).

5. R. Von Lindner and H. J. Matzke, "A Study of the Diffusion of $\mathrm{Xe}^{133}$ from Uranium Dioxide," Z. Naturforsch. 13A (1958), pp 794-796. (In German)

6. R. Von Lindner and H. J. Matzke, "Diffusion of $\mathrm{Xe}^{133}$ in Uranium Oxide of Various Oxygen Contents," Z. Naturforsch. 14A (1959), pp 582-584. (In German)

7. S. Flügge and K. E. Zimens, "Determination of Grain Sizes and Diffusion Constants from Emanation Power. A Theory of the Emanation Method," Z. physik. Chem., B42 (1939), pp $179-220$.

8. J. S. Anderson, E. A. Harper, S. Moorbath, and L. E. J. Roberts, "The Properties and Microstructure of Uranium Dioxide; Their Dependence upon the Mode of Preparation," AERE C/R 886 (1952).

9. J. Belle and B. Lustman, "Properties of $\mathrm{UO}_{2}$," in "Fuel Elements Conference, Paris, November 18-23, 1957, TID-7546, Book 2, pp 442-515.

\footnotetext{
*A. B. Auskern, unpublished data, Bettis Laboratory.

**W. A. Bostrom, personal communication, Bettis Laboratory.
} 